\title{
Controle Chaveado de Sistemas Lineares Incertos Sujeitos à Saturação no Atuador Usando Realimentação Derivativa
}

\author{
Leidy D. Wolmuth* Uiliam N. L. T. Alves ${ }^{* *}$ \\ Marcelo C. M. Teixeira ${ }^{* * *}$ Edvaldo Assunção*** \\ Rodrigo Cardim*** \\ * UFMT - Universidade Federal de Mato Grosso, ICET, Departamento \\ de Matemática, Cuiabá, Mato Grosso, (e-mail: wolmuth@gmail.com). \\ ** IFPR - Instituto Federal de Educação, Ciência e Tecnologia do \\ Paraná, Campus Jacarezinho, 86400-000, Jacarezinho, Paraná, \\ (e-mail: uiliam.alves@ifpr.edu.br) \\ *** Universidade Estadual Paulista (UNESP), Faculdade de \\ Engenharia, Ilha Solteira, SP, Brasil, (e-mails: \\ marcelo.minhoto@unesp.br,edvaldo.assuncao@unesp.br, \\ rodrigo.cardim@unesp.br)
}

\begin{abstract}
This paper considers a class of linear systems with time-invariant uncertainties, subject to actuator saturation, where only the derivative of the state vector is considered for feedback. In this scenario, the presented strategy uses an auxiliary dynamics, whose state is accessible for feedback, to control the original plant. It is proposed a switched control design procedure by means of linear matrix inequalities. If the conditions are feasible, they assure that the origin of the state space of the closed-loop system is locally asymptotically stable, for all initial for all initial conditions in an ellipsoidal region, which is within a given region defined for this new dynamics. Although the proposed design includes an auxiliary dynamics, it ensures the stability and decay rate proprieties for the original plant. A simulation example illustrates the effectiveness of the proposed approach.

Resumo: Neste artigo considera-se uma classe de sistemas lineares com incertezas invariantes no tempo, sujeito à saturação do atuador, onde apenas a derivada do vetor de estado é considerada para realimentação. Neste cenário, a estratégia proposta utiliza uma dinâmica auxiliar, cujo estado está disponível para realimentação, para controlar a planta original. Propõe-se um procedimento de projeto de controle chaveado por meio de desigualdades matriciais lineares. Se as condições forem satisfeitas, elas asseguram que a origem do espaço de estados do sistema em malha fechada é localmente assintoticamente estável, para todas as condições iniciais em uma região elipsoidal, que está dentro de uma determinada região definida para essa nova dinâmica. Embora o projeto proposto inclua uma dinâmica auxiliar, ele garante as propriedades de estabilidade e taxa de decaimento para a planta original. Um exemplo de simulação ilustra a eficácia da abordagem proposta.
\end{abstract}

Keywords: Derivative Feedback; Switched Control; Uncertain Linear Systems; Actuator Saturation; Theory of Control.

Palavras-chaves: Realimentação Derivativa; Controle Chaveado; Sistemas Lineares Incertos; Saturação do Atuador; Teoria de Controle.

\section{INTRODUÇÃO}

A realimentação derivativa pode ser útil no estudo de problemas práticos nos quais a derivada do vetor de estado é mais fácil de se medir do que o vetor de estado, por exemplo, no sistema de suspensão ativa de automóveis (Reithmeier and Leitmann, 2003; da Silva et al., 2013; Assunção et al., 2007). Em Yazici and Sever (2017b) é proposto um projeto de controladores com realimentação derivativa de saída para o controle ativo de vibração de um sistema de suspensão de veículos; um controle de vibrações em pontes suspensas por cabos com realimentação derivativa é encontrado em Duan et al. (2005); um projeto de controlador LQR de realimentação derivativa para um controle de vibração de uma plataforma offshore tipo jaqueta de aço é proposto em Yazici and Sever (2017a).

Projetos de controladores para sistemas mecânicos, incluindo o controle de vibrações, utilizando realimentação derivativa podem ser encontrados em Abdelaziz and Valás̆ek (2004), Abdelaziz and Valás̆ek (2005), Cardim et al. (2007) e Rossi et al. (2018).

Por outro lado, o controle chaveado pode ser usado para se obter um melhor desempenho em comparação ao uso 
de um ganho constante de realimentação na estrutura de controle. Nesse sentido, foi proposto em Souza et al. (2013) um controlador com ganho chaveado, para plantas lineares e invariantes no tempo com incertezas politópicas, que oferece uma alternativa menos conservadora ao uso de um ganho constante na realimentação do vetor estado. O procedimento proposto em Alves et al. (2016) também contempla uma região de operação e saturação do atuador no projeto do controlador chaveado. Além disso, de Oliveira et al. (2018) considera o índice de desempenho $\mathscr{H}_{\infty}$ nesta estrutura. É importante notar que todos os casos acima mencionados usam o vetor de estado para compor o sinal de controle. Em Moreira (2015), são apresentadas estratégias para utilizar o controle chaveado para uma classe de sistemas lineares incertos utilizando realimentação derivativa.

A saturação dos atuadores está presente em grande parte das aplicações práticas devido às restrições operacionais nos equipamentos. Em $\mathrm{Hu}$ et al. (2002) o domínio de atração da origem para um sistema linear saturado com realimentação do vetor de estado é estimado utilizando uma função quadrática de Lyapunov. Uma função de Lyapunov quadrática composta é apresentada em Hu and Lin (2003) para sistemas lineares contínuos no tempo com saturação, que mostram que, para um sistema linear saturado, a casca convexa de um conjunto de elipsóides invariantes é também invariante. Um projeto de controle chaveado é proposto em Alves et al. (2016) para uma classe de sistemas não lineares incertos, descritos por modelos fuzzy Takagi-Sugeno, dentro de uma região de operação no espaço de estados.

Tendo em vista o panorama apresentado, neste trabalho estabelecem-se condições para projeto de uma estrutura de controle chaveado baseada na realimentação derivativa apresentada em Wolmuth et al. (2019). Apresenta-se um exemplo no qual é comparado o uso da estrutura com ganho constante de realimentação (Wolmuth et al., 2019) e a estrutura com os ganhos chaveados, sendo que a proposta apresentada neste trabalho obteve melhores resultados. Os resultados numéricos, descritos ao longo do trabalho, foram obtidos utilizando o software MatLab ${ }^{\mathbb{R}}$ e o solver LMILab (Gahinet et al., 1994), interfaceado pelo YALMIP (Efberg and Löfberg, 2004), para resolver as condições de projeto e realizar as simulações.

Notações: $\mathfrak{R}$ representa o conjunto dos números reais, $Z_{+}$simboliza o conjunto dos números inteiros positivos, $\mathfrak{R}^{n}$ e $\mathfrak{R}^{n \times m}$ denotam o conjunto dos vetores $n \times 1$ com elementos reais e o conjunto das matrizes $n \times m$ com elementos reais, respectivamente. Define-se o conjunto $\mathbb{I}_{r}=\{1,2, \ldots, r\}, \quad r \in Z_{+}, \arg \min _{k \in \mathbb{I}_{r}}{ }^{*}\left\{h_{k}\right\}$ representa o menor índice $j \in \mathbb{I}_{r}$ tal que $h_{j}=\min _{k \in \mathbb{I}_{r}}\left\{h_{k}\right\}$. Denotase a combinação convexa dos vetores $w_{i}, \forall i \in \mathbb{I}_{r}$, por co $=\left\{w_{1}, w_{2}, \ldots, w_{r}\right\}$. A matriz de bloco diagonal formada pelas matrizes $M_{1}, M_{2}, \ldots, M_{r}$ é representada por $\operatorname{diag}\left\{M_{1}, M_{2}, \ldots, M_{r}\right\}, M_{(l)}$ representa a $l$-ésima linha de uma matriz $M, M>0(M<0, M \geq 0$ e $M \leq 0)$ significa que a matriz $M$ é definida positiva (definida negativa, semi-definida positiva, semi-definida negativa), respectivamente.

\section{CONTROLE DERIVATIVO COM SATURAÇÃO DO ATUADOR}

Considere o sistema linear com incertezas invariantes no tempo, sujeito à saturação no atuador, dado por:

$$
\dot{x}(t)=A(\alpha) x(t)+B \operatorname{sat}(u(t)),
$$

sendo $A(\alpha) \in \mathfrak{R}^{n \times n}$ e $B \in \mathfrak{R}^{n \times m}$ as matrizes que representam a dinâmica do sistema incerto, $x(t) \in \mathfrak{R}^{n}$ o vetor de estado e $u(t) \in \mathfrak{R}^{m}$ o vetor de entrada. A matriz $A(\alpha)$ é representada pela combinação convexa de vértices descrita por $A(\alpha)=$ $\sum_{i=1}^{r_{1}} \alpha_{i} A_{i}$, como feito em Boyd et al. (1994), com $\alpha \in \mathscr{P} \mathrm{e}$ $r_{1} \in Z_{+}$,

$$
\mathscr{P}=\left\{\alpha=\left[\begin{array}{lll}
\alpha_{1} & \ldots & \alpha_{r_{1}}
\end{array}\right]^{T}: \sum_{i=1}^{r_{1}} \alpha_{i}=1, \alpha_{i} \geq 0\right\} .
$$

A saturação do sinal de controle é dada por

$$
\begin{aligned}
\operatorname{sat}(u(t)) & =\left[\begin{array}{lll}
\operatorname{sat}\left(u_{1}(t)\right) & \cdots & \operatorname{sat}\left(u_{m}(t)\right)
\end{array}\right]^{T}, \\
\operatorname{sat}\left(u_{l}(t)\right) & =\operatorname{sgn}\left(u_{l}(t)\right) \min \left\{\rho_{l},\left|u_{l}(t)\right|\right\},
\end{aligned}
$$

$\operatorname{com} u(t)=\left[\begin{array}{lll}u_{1}(t) & \cdots & u_{m}(t)\end{array}\right]^{T}, \rho_{l}>0, \forall l \in \mathbb{I}_{m}$, um valor conhecido (Hu and Lin, 2003; Hu et al., 2002; Alves et al., 2016).

Dado o sistema linear incerto (1), considere que o posto de $A(\alpha)$, para todo $\alpha \in \mathscr{P}$, é completo. Então, tem-se que

$$
\begin{aligned}
\dot{x}(t) & =A(\alpha) x(t)+B \operatorname{sat}(u(t)) \\
A(\alpha) x(t) & =\dot{x}(t)-B \operatorname{sat}(u(t)) .
\end{aligned}
$$

Observe que, como a derivada do vetor de estado e a saturação do vetor de controle estão disponíveis, temos que $A(\alpha) x(t)$ também está disponível para realimentação. Agora, a partir de (3) é possível definir um novo vetor de estado do sistema (1) como feito por Moreira (2015); Wolmuth et al. (2019) tomando:

$$
\hat{x}(t)=A(\alpha) x(t) \text {. }
$$

De $(3)$ e $(4)$, temos que $\hat{x}(t)$ está disponível para realimentação pois tem-se acesso a $\dot{x}(t)$ e a $B$ sat $(u(t))$. Além disso, derivando (4) obtemos

$$
\dot{\hat{x}}(t)=A(\alpha) \dot{x}(t) .
$$

Pré-multiplicando (1) por $A(\alpha)$, e considerando (4) e (5),

$$
\dot{\hat{x}}(t)=A(\alpha) \hat{x}(t)+A(\alpha) B \operatorname{sat}(u(t)) .
$$

Observação 1. (Wolmuth et al., 2019) Para a realimentação derivativa estudada neste artigo, a suposição de que a matriz $A(\alpha)$ é uma matriz de posto completo para todo $\alpha$ definido em (2) é uma condição necessária para a estabilização da planta dada em (1) (Abdelaziz and Valášek, 2004; Assunção et al., 2007; Moreira et al., 2010; da Silva et al., 2011, 2012).

\subsection{Descrição da saturação do sinal de controle como combinação convexa}

Na Figura 1 é apresentada a representação gráfica da saturação do sinal de controle $u_{j}(t)$ e uma região especificada $\left|u_{j}(t)\right| \leq \phi_{j}$. 


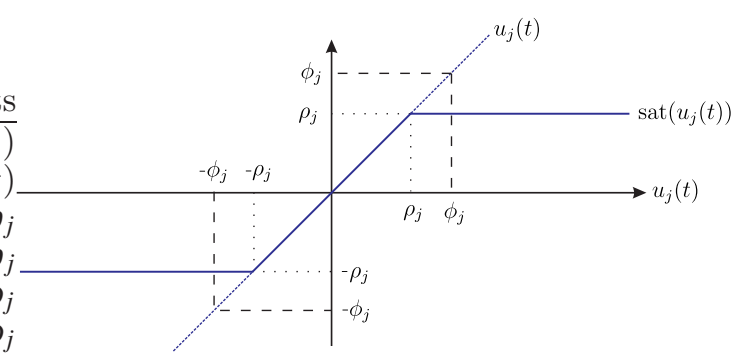

Figura 1. Representação de sat $\left(u_{j}(t)\right)$ como uma função de $u_{j}(t)$ e sua região de operação.

Dado o sistema linear incerto, assume-se que $\phi_{j}>0$ para todo $j \in \mathbb{I}_{m},-\phi_{j} \leq u_{j} \leq \phi_{j}$ e utiliza-se uma representação alternativa para a saturação de $u(t)$ dada em Wolmuth et al. (2019)

$$
\operatorname{sat}(u(t))=\left[\begin{array}{c}
\operatorname{sat}\left(u_{1}(t)\right) \\
\vdots \\
\operatorname{sat}\left(u_{m}(t)\right)
\end{array}\right], \operatorname{sat}\left(u_{j}(t)\right)=u_{j}(t) \varphi_{j}(t)
$$

com

$$
\varphi_{j}(t)=\left\{\begin{array}{r}
\left.1 \text { se } \mid u_{j}(t)\right) \mid \leq \rho_{j} \\
\rho_{j} /\left|u_{j}(t)\right| \text { se }\left|u_{j}(t)\right|>\rho_{j}
\end{array}\right.
$$

dessa forma $\max \left\{\varphi_{j}(t)\right\}=1$ e $\min \left\{\varphi_{j}(t)\right\}=\rho_{j} / \phi_{j}$. Assim, representa-se a função $\varphi_{j}(t)$ pela seguinte combinação convexa

$$
\varphi_{j}(t)=\tau_{1_{(j)}}(t) v_{1_{(j)}}+\tau_{2_{(j)}}(t) v_{2_{(j)}}
$$

com $v_{1_{(j)}}=1, v_{2_{(j)}}=\rho_{j} / \phi_{j}$. Observe que, $\tau_{1_{(j)}}(t)+\tau_{2_{(j)}}(t)=1$ e $\tau_{1_{(j)}}(t) \geq 0, \tau_{2_{(j)}}(t) \geq 0$, para todo $j \in \mathbb{I}_{m}$.

Pelo conhecimento dos autores, a descrição da saturação nessa forma foi proposta em Wolmuth et al. (2019).

Lema 1. (Wolmuth et al., 2019) Considere que existam constantes $\phi_{j}>0, j \in \mathbb{I}_{m}$, tais que $-\phi_{j} \leq u_{j} \leq \phi_{j}$ para todo $j \in \mathbb{I}_{m}$. Defina $Z_{s} \in \mathfrak{R}^{m \times m}, s \in \mathbb{I}_{2^{m}}$, as matrizes diagonais cujos elementos $(j, j)$ são todas as combinações possíveis de $v_{1_{(j)}}=1$ e $v_{2_{(j)}}=\rho_{j} / \phi_{j}$, para todo $j \in \mathbb{I}_{m}$. Então, de (7)-(9), obtemos:

$$
\operatorname{sat}(u(t))=Z(\lambda) u(t), \quad \text { com } \quad Z(\lambda)=\sum_{s=1}^{2^{m}} \lambda_{s} Z_{s}
$$

com o vetor incerto $\lambda \in \mathscr{L}$,

$$
\mathscr{L}=\left\{\lambda=\left[\begin{array}{lll}
\lambda_{1} & \ldots & \lambda_{2^{m}}
\end{array}\right]^{T}: \sum_{s=1}^{2^{m}} \lambda_{s}=1, \lambda_{s} \geq 0\right\} \text { e } 2^{m} \in Z_{+} .
$$

Prova. Veja Wolmuth et al. (2019)

Substituindo (10) em (6) obtém-se

$$
\dot{\hat{x}}(t)=A(\alpha) \hat{x}(t)+A(\alpha) B Z(\lambda) u(t) .
$$

Como $A(\alpha)=\sum_{i=1}^{r_{1}} \alpha_{i} A_{i}, Z(\lambda)=\sum_{s=1}^{2^{m}} \lambda_{s} Z_{s}, \sum_{s=1}^{2^{m}} \lambda_{s}=1 \mathrm{e}$ $\sum_{i=1}^{r_{1}} \alpha_{i}=1$, tomando $\mu_{k}=\lambda_{s} \alpha_{i} \operatorname{com} k=r_{1}(s-1)+(i-1)+1$ para todo $i \in \mathbb{I}_{r_{1}}$ e $s \in \mathbb{I}_{2^{m}}$ de (12) obtém-se

$$
\begin{aligned}
\dot{\hat{x}}(t)= & \sum_{s=1}^{2^{m}} \lambda_{s} \sum_{i=1}^{r_{1}} \alpha_{i} A_{i} \hat{x}(t)(t)+\sum_{i=1}^{r_{1}} \alpha_{i} A_{i} B \sum_{s=1}^{2^{m}} \lambda_{s} Z_{s} u(t) \\
= & \lambda_{1}\left\{\sum_{i=1}^{r_{1}} \alpha_{i} A_{i} \hat{x}(t)(t)+\sum_{i=1}^{r_{1}} \alpha_{i} A_{i} B Z_{1} u(t)\right\}+\cdots \\
& +\lambda_{2^{m}}\left\{\sum_{i=1}^{r_{1}} \alpha_{i} A_{i} \hat{x}(t)(t)+\sum_{i=1}^{r_{1}} \alpha_{i} A_{i} B Z_{2^{m}} u(t)\right\} \\
= & \lambda_{1}\left\{\alpha_{1}\left\{A_{1} \hat{x}(t)(t)+A_{1} B Z_{1} u(t)\right\}\right\} \\
& +\cdots+\lambda_{1}\left\{\alpha_{r_{1}}\left\{A_{r_{1}} \hat{x}(t)(t)+A_{r_{1}} B Z_{1} u(t)\right\}\right\} \\
& +\cdots+\lambda_{2^{m}}\left\{\alpha_{1}\left\{A_{1} \hat{x}(t)(t)+A_{1} B Z_{2^{m}} u(t)\right\}\right\} \\
& +\cdots+\lambda_{2^{m}}\left\{\alpha_{r_{1}}\left\{A_{r_{1}} \hat{x}(t)(t)+A_{r_{1}} B Z_{2^{m}} u(t)\right\}\right\} \\
= & \mu_{1}\left\{A_{1} \hat{x}(t)(t)+A_{1} B Z_{1} u(t)\right\} \\
& +\cdots+\mu_{r_{1}}\left\{A_{r_{1}} \hat{x}(t)(t)+A_{r_{1}} B Z_{1} u(t)\right\} \\
& +\mu_{r_{1}+1}\left\{A_{1} \hat{x}(t)(t)+A_{1} B Z_{2} u(t)\right\} \\
& +\cdots+\mu_{2 r_{1}}\left\{A_{r_{1}} \hat{x}(t)(t)+A_{r_{1}} B Z_{2} u(t)\right\} \\
& +\cdots+\mu_{\left(2^{m}-1\right) r_{1}+1}\left\{A_{1} \hat{x}(t)(t)+A_{1} B Z_{2^{m}} u(t)\right\} \\
& +\cdots+\mu_{2^{m} r_{1}}\left\{A_{r_{1}} \hat{x}(t)(t)+A_{r_{1}} B Z_{2^{m}} u(t)\right\},
\end{aligned}
$$

com $k \in \mathbb{I}_{r}, \quad r=2^{m} r_{1}$ o número de incertezas do sistema (12), $\sum_{k=1}^{r} \mu_{k}=1$ e $\mu_{k} \geq 0$.

Em (13) temos que:

$\hat{A}_{f r_{1}+k}=A_{k}$, para todo $k \in\left\{1, \ldots, r_{1}\right\}$ e para cada $f \in\left\{0,1, \ldots, 2^{m}-1\right\}$,

$$
\begin{aligned}
\hat{Z}_{k}= & Z_{s} \text { para } k=(s-1) r_{1}+1, \cdots, s r_{1} \quad \text { e para cada } \\
& s \in\left\{1, \ldots, 2^{m}\right\} \\
\hat{B}_{k}= & \hat{A}_{k} B \hat{Z}_{k} \text { para todo } k \in\{1, \ldots, r\} .
\end{aligned}
$$

Assim, definindo-se $\mu=\left[\begin{array}{llll}\mu_{1} & \mu_{2} & \ldots & \mu_{r}\end{array}\right]^{T}$,

$$
\dot{\hat{x}}(t)=\sum_{k=1}^{r} \mu_{k}\left\{\hat{A}_{k} \hat{x}(t)+\hat{B}_{k} u(t)\right\}
$$

Ou equivalentemente,

$$
\dot{\hat{x}}(t)=\hat{A}(\mu) \hat{x}(t)+\hat{B}(\mu) u(t) .
$$

\subsection{Controle Robusto Chaveado para a Dinâmica Auxiliar}

Considere o esquema de controle proposto na Figura 2. A partir deste diagrama, a lei de controle robusta chaveada é dada por

$$
\begin{aligned}
& u_{N}(t)=u_{N_{\sigma}}(t)=\dot{u}(t)=-K_{\sigma} x_{N}(t), \\
& \sigma=\arg \min _{k \in \mathbb{I}_{r}} *\left\{x_{N}(t)^{T} P B_{N}\left(-K_{k}\right) x_{N}(t)\right\}, \text { com } \\
& \sigma \in \mathbb{I}_{r}, K_{\sigma} \in \mathfrak{R}^{m \times(n+m)} \text { e } x_{N}(t) \in \mathfrak{R}^{n+m} \text {. }
\end{aligned}
$$

De (16) e (17) tem-se o seguinte sistema:

$$
\begin{gathered}
\left\{\begin{array}{l}
\dot{\hat{x}}(t)=\hat{A}(\mu) \hat{x}(t)+\hat{B}(\mu) u(t), \\
\dot{u}=u_{N}(t)=-K_{\sigma} x_{N}(t),
\end{array}\right. \\
\dot{x}_{N}(t)=\sum_{k=1}^{r} \mu_{k} A_{N_{k}}(t) x_{N}(t)+\sum_{k=1}^{r} \mu_{k} B_{N} u_{N}(t),
\end{gathered}
$$




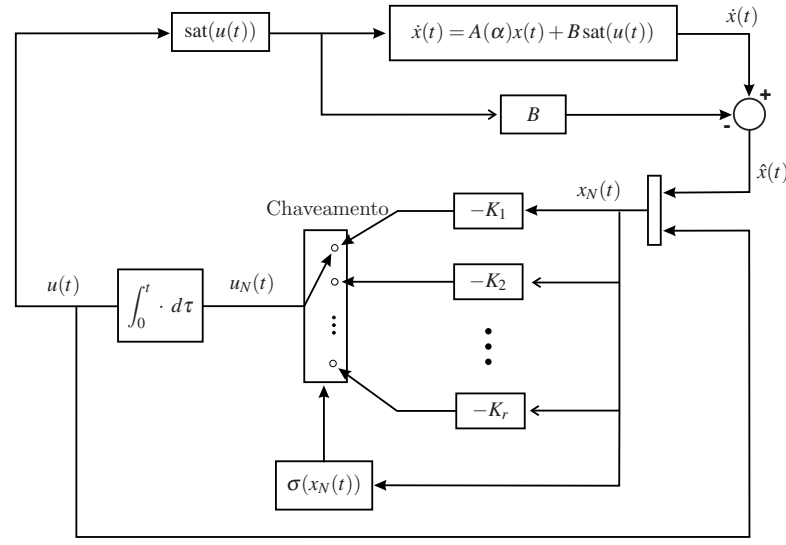

Figura 2. Esquema da lei de controle proposta (17) para o sistema linear incerto sujeito à saturação do atuador (1).

$$
A_{N_{k}}=\left[\begin{array}{cc}
\hat{A}_{k} & \hat{B}_{k} \\
0_{m \times n} & 0_{m \times m}
\end{array}\right], \quad x_{N}(t)=\left[\begin{array}{c}
\hat{x}(t) \\
u(t)
\end{array}\right],
$$

$B_{N}=\left[\begin{array}{ll}0_{m \times n} & I_{m \times m}\end{array}\right]^{T}, \hat{A}_{k}$ e $\hat{B}_{k}$, definidos em (14), ou equivalentemente

$$
\dot{x}_{N}(t)=A_{N}(\mu)(t) x_{N}(t)+B_{N} u_{N}(t) .
$$

É interessante notar que o sinal de controle $u(t)$ compõe o vetor de estado $x_{N}(t)$, portanto a restrição $\left|u_{j}(t)\right| \leq$ $\phi_{j}$, utilizada para obter (20), pode ser vista como uma região de operação para o dinâmica auxiliar. Assim, considere matrizes $N=\left[\begin{array}{ll}0_{m \times n} & I_{m \times m}\end{array}\right] \in \Re^{m \times(n+m)}, \quad \phi=$ $\left[\begin{array}{llll}\phi_{1} & \phi_{2} & \ldots & \phi_{m}\end{array}\right]^{T} \in \mathfrak{R}^{m}$ e $P=P^{T} \in \mathfrak{R}^{(n+m) \times(n+m)}, P>0$, um vetor $\rho=\left[\begin{array}{lll}\rho_{1} & \ldots & \rho_{m}\end{array}\right]^{T} \in \mathfrak{R}^{m}, \mathbb{I}_{m}=\{1,2, \cdots, m\}, \rho_{j}>$ $0, \phi_{j} \geq \rho_{j}$, para todo $j \in \mathbb{I}_{m}$ e uma constante positiva $\delta$.

Sejam $\mathscr{X}_{u}$ e $\mathscr{E}(P, \delta)$ os seguintes conjuntos:

$$
\mathscr{X}_{u} \triangleq\left\{x_{N}(t) \in \mathfrak{R}^{n+m}:\left|N_{(h)} x_{N}(t)\right| \leq \phi_{h}, h \in \mathbb{I}_{m}\right\},
$$

com $N=\left[\begin{array}{ll}0_{m \times n} & I_{m \times m}\end{array}\right]$,

$$
\mathscr{E}(P, \delta) \triangleq\left\{x_{N}(t) \in \mathfrak{R}^{n+m}: x_{N}(t)^{T} P x_{N}(t) \leq \delta\right\}
$$

com $N$ e $\phi$ conhecidas. $N_{(h)}$ representa a linha $h$ da matriz $N$ e $\phi_{h}$ a entrada $h$ do vetor $\phi$ (Klug et al., 2015; Alves et al., 2016; de Oliveira et al., 2018).

Teorema 1. Considere o sistema linear sujeito à saturação no atuador descrito em (18)-(20) com a lei de controle (17) e uma região de operação com $x_{N}(t) \in \mathscr{X}_{u}, t \geq 0$ dada em (21) sendo $\phi>0 \in \mathfrak{R}^{m}, N=\left[\begin{array}{ll}0_{m \times n} & I_{m \times m}\end{array}\right] \in \mathfrak{R}^{m \times(n+m)} \mathrm{e}$ $\rho>0 \in \mathfrak{R}^{m}$ conhecidos. Suponha que existem uma matriz simétrica definida positiva $X \in \Re^{(n+m) \times(n+m)}$, matrizes $M_{k} \in$ $\mathfrak{R}^{m \times(m+n)}$ e um escalar $\beta>0$, tais que

$$
\begin{gathered}
A_{N_{k}} X+X A_{N_{k}}^{T}-B_{N} M_{k}-M_{k}^{T} B_{N}^{T}+2 \beta X<0, \\
{\left[\begin{array}{cc}
\phi_{h}^{2} & N_{(h)} X \\
X N_{(h)}^{T} & X
\end{array}\right] \geq 0,}
\end{gathered}
$$

para todo $h \in \mathbb{I}_{m}, k \in \mathbb{I}_{r}$. Então, a lei de controle (17), com $K_{k}=M_{k} X^{-1}$, torna a origem do espaço de estados do sistema (20) localmente assintoticamente estável com taxa de decaimento maior ou igual a $\beta$ para todo $x_{N}(0) \in \mathscr{E}(P, 1)$ dado em (22) sendo que $\delta=1$ e $P=X^{-1}$.

Prova. Considere a candidata a função de Lyapunov $V\left(x_{N}(t)\right)=x_{N}(t)^{T} P x_{N}(t)$, sendo $P=P^{T}>0$. Defina $X=P^{-1}$,
$M_{k}=K_{k} X$ e considere as LMIs descritas em (23) e (24), para todo $h \in \mathbb{I}_{m}, k \in \mathbb{I}_{r}$, factíveis.

Então de $(23)$ e $M_{k}=K_{k} X$

$$
\begin{aligned}
0 & >A_{N_{k}} X+X A_{N_{k}}{ }^{T}-B_{N} M_{k}-M_{k}^{T} B_{N}^{T}+2 \beta X \\
& =A_{N_{k}} X+X A_{N_{k}}{ }^{T}-B_{N} K_{k} X-X{K_{k}}^{T} B_{N}^{T}+2 \beta X .
\end{aligned}
$$

Pré e pós-multiplicando (25) por $P=P^{T}>0$

$$
0>P A_{N_{k}}+A_{N_{k}}^{T} P-P B_{N} K_{k}-K_{k}^{T} B_{N}^{T} P+2 \beta P .
$$

Supondo $x_{N}(t) \neq 0$, pré e pós-multiplicando $(26)$ por $x_{N}(t)^{T}$ e $x_{N}(t)$, respectivamente, tem-se

$$
\begin{aligned}
0> & x_{N}(t)^{T}\left(P A_{N_{k}}+A_{N_{k}}^{T} P\right) x_{N}(t)+2 \beta x_{N}(t)^{T} P x_{N}(t) \\
& -x_{N}(t)^{T}\left(P B_{N} K_{k}+K_{k}^{T} B_{N}^{T} P\right) x_{N}(t) \\
= & 2 x_{N}(t)^{T} P A_{N_{k}} x_{N}(t)+2 \beta x_{N}(t)^{T} P x_{N}(t) \\
& +2 x_{N}(t)^{T} P B_{N}\left(-K_{k}\right) x_{N}(t) .
\end{aligned}
$$

Multiplicando (27) por $\mu_{k}, k \in \mathbb{I}_{r}$ e somando todos os termos para $k=1$ até $r$, de $\sum_{k=1}^{r} \mu_{k}=1$ e (20),

$$
\begin{aligned}
0> & \sum_{k=1}^{r} \mu_{k}\left(2 x_{N}(t)^{T} P A_{N_{k}} x_{N}(t)+2 \beta x_{N}(t)^{T} P x_{N}(t)\right) \\
& +2 x_{N}(t)^{T} P B_{N}\left(-\sum_{k=1}^{r} \mu_{k} K_{k}\right) x_{N}(t) .
\end{aligned}
$$

De $\sum_{k=1}^{r} \mu_{k}=1, \mu_{k} \geq 0, k \in \mathbb{I}_{r}$, note que de (17),

$$
\begin{aligned}
x_{N}(t)^{T} P B_{N}\left(-K_{\sigma}\right) x_{N}(t) & =\min _{k \in \mathbb{I}_{r}}\left\{x_{N}(t)^{T} P B_{N}\left(-K_{k}\right) x_{N}(t)\right\} \\
& \leq x_{N}(t)^{T} P B_{N}\left(-\sum_{k=1}^{r} \mu_{k} K_{k}\right) x_{N}(t) .
\end{aligned}
$$

De (17), (28) e (29), tem-se

$$
\begin{aligned}
0> & 2 x_{N}(t)^{T} P A_{N}(\mu) x_{N}(t)+2 \beta x_{N}(t)^{T} P x_{N}(t) \\
& +2 x_{N}(t)^{T} P B_{N}\left(-\sum_{k=1}^{r} \mu_{k} K_{k}\right) x_{N}(t) \\
\geq & 2 x_{N}(t)^{T} P A_{N}(\mu) x_{N}(t)+2 \beta x_{N}(t)^{T} P x_{N}(t) \\
& +2 x_{N}(t)^{T} P B_{N}\left(-K_{\sigma}\right) x_{N}(t) \\
= & 2 x_{N}(t)^{T} P\left[A_{N}(\mu) x_{N}(t)+B_{N} u_{N}(t)\right] \\
& +2 \beta x_{N}(t)^{T} P x_{N}(t) \\
= & 2 x_{N}(t)^{T} P \dot{x}_{N}(t)+2 \beta x_{N}(t)^{T} P x_{N}(t) \\
= & \dot{V}\left(x_{N}(t)\right)+2 \beta V\left(x_{N}(t)\right) .
\end{aligned}
$$

Portanto, $\dot{V}\left(x_{N}(t)\right)<-2 \beta V\left(x_{N}(t)\right)$ e a origem do sistema em malha fechada (20) e (17) é localmente assintoticamente estável com taxa de decaimento igual ou maior que $\beta$ (Boyd et al., 1994).

Pré e pós-multiplicando a LMI (24) por $\operatorname{diag}\{1, P\}$ com $P=X^{-1}$, observe que

$$
\left[\begin{array}{cc}
\phi_{h}^{2} & N_{(h)} \\
N_{(h)} & P
\end{array}\right] \geq 0
$$


Aplicando o Complemento de Schur (Boyd et al., 1994) em (30), tem-se

$$
P-N_{(h)}^{T} \phi_{h}{ }^{-2} N_{(h)} \geq 0 .
$$

Pré-multiplicando (31) por $x_{N}(t)^{T}$ e pós-multiplicando por $x_{N}(t)$, encontra-se a seguinte desigualdade:

$$
x_{N}(t)^{T} P x_{N}(t) \geq x_{N}(t)^{T} N_{(h)}^{T} \phi_{h}{ }^{-2} N_{(h)} x_{N}(t) .
$$

Se $x_{N}(t) \in \mathscr{E}(P, 1)$ definido em $(22)$, então $x_{N}(t)^{T} P x_{N}(t) \leq 1$,

$$
\begin{aligned}
\phi_{h}{ }^{2} & \geq x_{N}(t)^{T} N_{(h)}^{T} N_{(h)} x_{N}(t)=\left|N_{(h)} x_{N}(t)\right|^{2} \\
& \Rightarrow\left|N_{(h)} x_{N}(t)\right| \leq \phi_{h} \quad \forall \quad h \in \mathbb{I}_{m} .
\end{aligned}
$$

Então, se (24) é factível, para todo $x_{N}(t) \in \mathscr{E}(P, 1)$ segue que $\mathscr{E}(P, 1) \subset \mathscr{X}_{u}$. Como $\dot{V}\left(x_{N}(t)\right)<0$ para $x_{N}(t) \neq 0$, de (22) se $x_{N}(0) \in \mathscr{E}(P, 1)$, desde que (24) seja factível para todo $h \in \mathbb{I}_{m}$, então $x_{N}(t) \in \mathscr{E}(P, 1)$ para todo $t \geq 0$.

A lei de controle dada por $u_{N}(t)=\dot{u}(t)=-K x_{N}(t)$ é uma alternativa para resolver o problema de controle local. Usando a candidata a função de Lyapunov $V\left(x_{N}(t)\right)=$ $x_{N}(t)^{T} P x_{N}(t)$, as condições de projeto de controle podem ser enunciadas de maneira similar ao Teorema 1.

Corolário 1. (Wolmuth et al., 2019) Considere o sistema linear sujeito à saturação no atuador descrito em (20) com lei de controle dada por $u_{N}(t)=-K x_{N}(t)$ e uma região de operação com $x_{N}(t) \in \mathscr{X}_{u}, t \geq 0$ dada em (21) sendo que $\phi>0 \in \mathfrak{R}^{m}, N=\left[\begin{array}{ll}0_{m \times n} & I_{m \times m}\end{array}\right] \in \mathfrak{R}^{m \times(n+m)}$ e $\rho>0 \in \mathfrak{R}^{m}$ conhecidos. Assuma que existem uma matriz simétrica definida positiva $X \in \mathfrak{R}^{(n+m) \times(n+m)}$, uma matriz $M \in \mathfrak{R}^{m \times(m+n)}$ e um escalar $\beta>0$, tais que

$$
\begin{gathered}
A_{N_{k}} X+X A_{N_{k}}^{T}-B_{N} M-M^{T} B_{N}^{T}+2 \beta X<0, \\
{\left[\begin{array}{cc}
\phi_{h}^{2} & N_{(h)} X \\
X N_{(h)}^{T} & X
\end{array}\right] \geq 0,}
\end{gathered}
$$

para todo $h \in \mathbb{I}_{m}, k \in \mathbb{I}_{r}$. Então, a lei de controle $u_{N}(t)=$ $-K x_{N}(t)$, com $K=M X^{-1}$, torna a origem do espaço de estados do sistema (20) localmente assintoticamente estável com taxa de decaimento maior ou igual a $\beta$ para todo $x_{N}(0) \in \mathscr{E}(P, 1)$ dado em $(22)$ sendo que $\delta=1$ e $P=X^{-1}$.

Prova. Veja Wolmuth et al. (2019).

Para as dinâmicas propostas com $x_{N}(t)=\left[\hat{x}(t)^{T} u(t)^{T}\right]^{T}$, $\operatorname{sat}(u(t)), u_{N}(t)=\dot{u}(t)$ e ganhos de realimentação $K$ e $K_{k}$, a saturação de $u(t)$ garante uma restrição para a entrada $u(t)$. A utilização das LMIs para reduzir a norma dos ganhos da realimentação $K$ e $K_{k}$, justifica-se devido às leis de controle $u_{N}(t)=-K x_{N}(t)$ e $u_{N}(t)=-K_{\sigma} x_{N}(t)$ (17) serem baseadas em $u_{N}(t)=\dot{u}(t)$.

Teorema 2. (Assunção et al., 2007; Buzetti, 2017) Dada uma constante $\mu_{0}>0$, então a norma dos ganhos de realimentação $K_{k}, \quad k \in \mathbb{I}_{r}$, pode ser majorada utilizando a minimização de $\eta, \eta>0$, tal que $K_{k} K_{k}^{T}<\eta I / \mu_{0}{ }^{2}$. O valor ótimo de $\eta$ pode ser obtido solucionando o seguinte problema de otimização:

$$
\begin{gathered}
\min \eta \\
{\left[\begin{array}{cc}
\eta I & M_{k} \\
M_{k}^{T} & I
\end{array}\right]>0}
\end{gathered}
$$

$$
X>\mu_{0} I
$$

sendo que o conjunto de LMIs pode ser igual a (23), (24) ou (34), (35) (substituindo $M_{k}$ por $M$ em (36)).

Prova. Veja Assunção et al. (2007).

O Teorema 1 e o Corolário 1 garantem a estabilidade local, com taxa de decaimento maior ou igual a $\beta$, para a origem do sistema linear incerto (20) em malha fechada, com a lei de controle $(17)$ e $u_{N}(t)=-K x_{N}(t)$, respectivamente, para qualquer condição inicial $x_{N}(0) \in \mathscr{E}(P, 1)$. Em Wolmuth et al. (2019) é garantida uma taxa de decaimento para o sistema (1) com vetor de estado $x(t)$; mostra-se que $\|x(t)\|$ é limitada para $t \geq 0$ e para toda trajetória $x(t)$, é garantida a existência de uma taxa de decaimento maior ou igual a $\beta$.

Da mesma forma que em Alves et al. (2016) e Wolmuth et al. (2019), consideram-se condições iniciais politópicas, o vetor de estado da planta $x(0) \in \mathscr{X}_{0}$, com $\mathscr{X}_{0}=\operatorname{co}\left\{x_{0_{1}}, \ldots, x_{0_{q}}\right\}, x_{0_{e}} \in \mathfrak{R}^{n}$ para todo $e \in \mathbb{I}_{q}, x(0)=$ $\sum_{e=1}^{q} \eta_{e} x_{0_{e}}, \eta_{e} \geq 0$ e $\sum_{e=1}^{q} \eta_{e}=1$, que é o casco convexo dos vetores conhecidos $x_{0_{1}}, \ldots, x_{0_{q}}$. A partir de $\mathscr{X}_{0}$, definese os conjuntos de condições iniciais de interesse para o vetor de estado $x(t)$ e para $x_{N}(t)=\left[\begin{array}{ll}(A(\alpha) x(t))^{T} & u(t)^{T}\end{array}\right]^{T}$, respectivamente como $\hat{w} \mathscr{X}_{0}$ e $\hat{w} \mathscr{W} \in \mathscr{E}(P, 1)$, com $\hat{w}>0$, $u(0)=0_{m \times 1}$, sendo $\mathscr{W}=\operatorname{co}\left\{\left[\begin{array}{c}A(\alpha) x_{0_{1}} \\ 0_{m \times 1}\end{array}\right], \ldots,\left[\begin{array}{c}A(\alpha) x_{0_{q}} \\ 0_{m \times 1}\end{array}\right]\right\}$, o casco convexo de vetores $\left[\begin{array}{c}A(\alpha) x_{0_{1}} \\ 0_{m \times 1}\end{array}\right], \ldots,\left[\begin{array}{c}A(\alpha) x_{0_{q}} \\ 0_{m \times 1}\end{array}\right]$, $\operatorname{com} \sum_{e=1}^{q} \eta_{e}\left[\begin{array}{c}A(\alpha) x_{0_{e}} \\ 0_{m \times 1}\end{array}\right] \in \mathscr{W}, \sum_{e=1}^{q} \eta_{e}=1, \eta_{e} \geq 0$ para todo $e \in \mathbb{I}_{q}$, com o vetor incerto mas constante $\alpha \in \mathscr{P}(2)$.

Lema 2. (Wolmuth et al., 2019) A condição $\hat{w} \mathscr{W} \subset \mathscr{E}(P, 1)$ é satisfeita se

$$
\left[\begin{array}{cc}
\hat{w}^{-2} & {\left[\begin{array}{c}
A_{i} x_{0_{e}} \\
0_{m \times 1}
\end{array}\right]^{T}} \\
{\left[\begin{array}{c}
A_{i} x_{0_{e}} \\
0_{m \times 1}
\end{array}\right]} & X
\end{array}\right] \geq 0
$$

com $\hat{w}$ uma constante positiva, para todo $e \in \mathbb{I}_{q}$ e $i \in$ $\mathbb{I}_{r_{1}}$. Assim $\hat{w}$ pode ser usado como uma variável para se obter uma estimativa menos conservadora do domínio de atração na busca do maior elipsóide $\mathscr{E}(P, 1)$ que contenha as condições iniciais de interesse (Alves et al., 2016; Hu et al., 2002; Cao and Lin, 2003).

Prova. Veja Wolmuth et al. (2019).

O teorema a seguir apresenta uma análise teórica da estabilidade entre o procedimento proposto no Teorema 1 , que usa a lei de controle chaveada, com o procedimento apresentado no Corolário 1, que usa uma lei de controle dada por $u_{N}(t)=-K x_{N}(t)$.

Teorema 3. Se as condições dadas no Corolário 1 com lei de controle $u_{N}(t)=-K x_{N}(t)$ são factíveis, então as condições dadas no Teorema 1 com a lei de controle chaveada (17) também são factíveis.

Prova. Segue passos semelhantes aos apresentados em Buzetti (2017). 


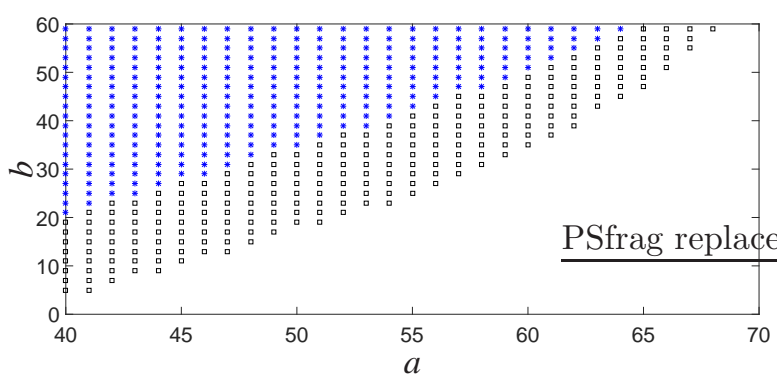

Figura 3. Região de factibilidade para $\hat{w}=0,2236$ no Lema $2, \eta=6,7968 \times 10^{6}$ e $\mu_{0}=1$ no Teorema 2: com Corolário 1 representado por $(*)$ e com Teorema 1 representado por $(*, \square)$.

\section{EXEMPLO}

Nesta seção, apresentamos um exemplo de simulação baseado em Moreira (2015) e Wolmuth et al. (2019) para ilustrar a eficácia da abordagem proposta. São descritas comparações entre o controle robusto com ganho constante usando a estratégia prosposta em Wolmuth et al. (2019) e a proposta apresentada neste artigo com ganhos chaveados.

Considere o sistema linear incerto (1) com vértices politópicos:

$$
A(\alpha)=\left[\begin{array}{cc}
\check{a} & -100 \\
10 & \check{b}
\end{array}\right], \quad B=\left[\begin{array}{l}
10 \\
10
\end{array}\right]
$$

com $-1 \leq \check{a} \leq a$ e $b \leq \breve{b} \leq 110$. Os vértices do politopo de $A(\alpha)$ são dados por:

$$
\left[\mathbf{A}_{1}\left|\mathbf{A}_{2}\right| \mathbf{A}_{3} \mid \mathbf{A}_{4}\right]=\left[\begin{array}{cc|cc|cc|cc}
-1 & -100 & a & -100 & -1 & -100 & a & -100 \\
10 & 110 & 10 & 110 & 10 & b & 10 & b
\end{array}\right]
$$

com $a$ e $b$ variando nos intervalos $40 \leq a \leq 90$ e $-9 \leq$ $b \leq 60$. Para que o sistema (1) esteja na forma adequada para a utilização do Teorema 1 e Corolário 1, construindo a dinâmica auxiliar (18)-(20) tem-se

$$
A_{N_{k}}=\left[\begin{array}{cc}
\hat{A}_{k} & \hat{A}_{k} B Z_{1} \\
0_{1 \times 2} & 0_{1 \times 1}
\end{array}\right] k=1, \ldots, 4, A_{N_{k}}=\left[\begin{array}{cc}
\hat{A}_{k} & \hat{A}_{k} B Z_{2} \\
0_{1 \times 2} & 0_{1 \times 1}
\end{array}\right] k=5, \ldots, 8,
$$

$\operatorname{com} B_{N}=\left[\begin{array}{lll}0 & 0 & 1\end{array}\right]^{T}, x_{N}(t)=\left[\begin{array}{ll}\hat{x}(t)^{T} u(t)^{T}\end{array}\right]^{T}, Z_{1}=1, Z_{2}=\frac{\rho}{\phi}$, $\hat{A}_{5}=\hat{A}_{1}=A_{1}, \hat{A}_{6}=\hat{A}_{2}=A_{2}, \hat{A}_{7}=\hat{A}_{3}=A_{3}$ e $\hat{A}_{8}=\hat{A}_{4}=A_{4}$, com taxa de decaimento $\beta=2,44$ para as LMIs (38) presentes no Lema $2, \operatorname{com} q=2, x_{0_{1}}=\left[\begin{array}{ll}0,1 & 0,0\end{array}\right]^{T}$ e $x_{0_{2}}=$ $\left[\begin{array}{ll}-0,1 & 0,0\end{array}\right]^{T}, \phi=1,2, \rho=1$ e $\mu_{0}=1$.

O parâmetro $\eta$ foi escolhido através de um procedimento de busca, visando aumentar a região factível e ao mesmo tempo maximizar o parâmetro $\hat{w}$. Para obter a região de factibilidade da Figura 3, escolheu-se de forma adequada $\bar{w}=20,0016 \operatorname{com} \hat{w}=\bar{w}^{-1 / 2}=0,2236, \eta=6,7968 \times 10^{6}$, com espaçamento uniforme de 1 para $a$ e de 2 para $b$, para ilustrar a comparação proposta no Teorema 3 .

Observe que as regiões obtidas nas Figuras 3 e 4, para os Teorema 1 com o Teorema 2 e Lema 2 representada por $(*, \square)$ e (-) é maior que a obtida pelo Corolário 1 , Teorema 2 (tomando $K_{k}=K$ ) e Lema 2 representada por (*) e (-), respectivamente. Além disso, na Figura 4 podese observar que os valores de $\hat{w}$ obtidos para o controle chaveado são maiores que os obtidos para o uso de um único ganho de realimentação. Isto se reflete na garantia

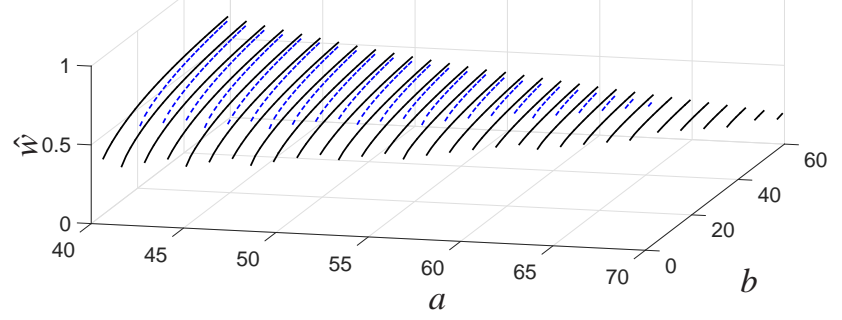

Figura 4. Região de factibilidade obtida minimizando $\bar{w}$ com $\bar{w}=\hat{w}^{-2}$, o que resulta na maximização de $\hat{w}$ no Lema 2, mantendo $\eta=6,7968 \times 10^{6}$ fixo e $\mu_{0}=1$ no Teorema 2: com Corolário 1 representado por (- -) e com o Teorema 1 representado por (-).

de estabilidade local para uma região elipsoidal maior para o ganho chaveado do que para único ganho.

Considere o ponto da região de factibilidade $(a, b)=$ $(60,50)$ da Figura 3. Para o Corolário 1, Teorema 2 (tomando $K_{k}=K$ ) e Lema 2 , minimiza-se $\bar{w} \operatorname{com} \bar{w}=\hat{w}^{-2}$, o que resulta na maximização de $\hat{w}$, obtém-se $\hat{w}=0,2236$, para $\eta=6,7968 \times 10^{6}$ e $\bar{\mu}_{0}=1$,

$$
\begin{aligned}
& K=10^{3}[-0,75150,41432,3752] \text {, } \\
& P=\left[\begin{array}{ccc}
0,5611 & -0,0287 & -0,2027 \\
-0,0287 & 0,1439 & 0,1368 \\
-0,2027 & 0,1368 & 0,8800
\end{array}\right] \text {, } \\
& \|K\|=2,5255 \times 10^{3},\|P\|=1,0000 \text {. }
\end{aligned}
$$

Para a solução, minimiza-se $\bar{w}$ com $\bar{w}=\hat{w}^{-2}$ com as LMIs do Lema 2 e Teoremas 1 e 2, obtém-se $\hat{w}=0,2950$, mantémse fixo $\eta=6,7968 \times 10^{6}$ e $\mu_{0}=1$,

$$
\begin{aligned}
K_{1} & =10^{3}\left[\begin{array}{lll}
-0,4511 & 0,3381 & 1,6030
\end{array}\right], \\
K_{2} & =10^{3}\left[\begin{array}{lll}
-0,3653 & 0,3367 & 1,5508
\end{array}\right], \\
K_{3} & =10^{3}\left[\begin{array}{lll}
-0,4867 & 0,3023 & 1,5444
\end{array}\right], \\
K_{4} & =10^{3}\left[\begin{array}{lll}
-0,3919 & 0,3115 & 1,5083
\end{array}\right], \\
K_{5} & =10^{3}\left[\begin{array}{lll}
-0,4250 & 0,3369 & 1,5708
\end{array}\right], \\
K_{6} & =10^{3}\left[\begin{array}{lll}
-0,3635 & 0,3364 & 1,5257
\end{array}\right], \\
K_{7} & =10^{3}\left[\begin{array}{lll}
-0,4553 & 0,3034 & 1,5308
\end{array}\right], \\
K_{8} & =10^{3}\left[\begin{array}{lll}
-0,3810 & 0,3088 & 1,4958
\end{array}\right], \\
P & =\left[\begin{array}{ccc}
0,3351 & -0,0579 & -0,1637 \\
-0,0579 & 0,1182 & 0,1565 \\
-0,1637 & 0,1565 & 0,9264
\end{array}\right], \\
\left\|K_{1}\right\| & =1,6992 \times 10^{3},\left\|K_{2}\right\|=1,6284 \times 10^{3}, \\
\left\|K_{3}\right\| & =1,6472 \times 10^{3},\left\|K_{4}\right\|=1,5892 \times 10^{3}, \\
\left\|K_{5}\right\| & =1,6618 \times 10^{3},\left\|K_{6}\right\|=1,6041 \times 10^{3}, \\
\left\|K_{7}\right\| & =1,6256 \times 10^{3},\left\|K_{8}\right\|=1,5742 \times 10^{3}, \\
\|P\| & =1,0000 .
\end{aligned}
$$

Para as simulações, considere a condição inicial $x(0)=$ $A_{2}{ }^{-1}[1,081,76]^{T}$ e o ponto da região de factibilidade $(a, b)=(60,50)$ da Figura 3 . 


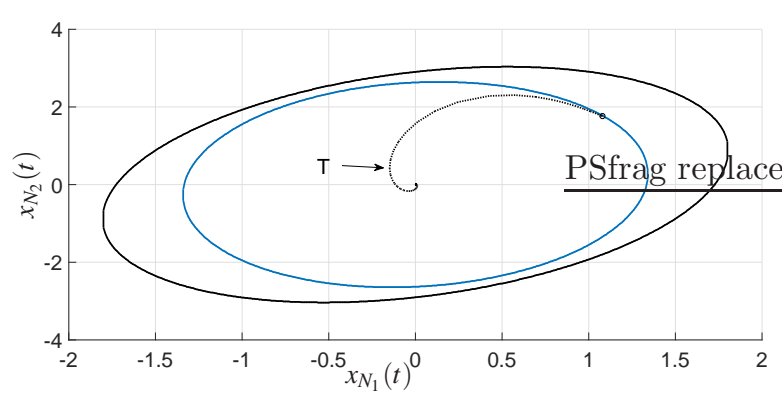

Figura 5. Região elipsoidal $\mathscr{E}(P, 1)$ e trajetória para $x_{N}(t)=$ $\left[\hat{x}(t)^{T} \quad u(t)^{T}\right]^{T} \operatorname{com} x_{N_{3}}(t)=u(t)=0,(\mathrm{a}, \mathrm{b})=(60,50)$ da Figura 3, Teorema 2 e Lema 2: com o Teorema 1 representado por (-); o Corolário 1 representado por (-); a curva $T$ representa a trajetória para a condição inicial $x(0)=A_{2}^{-1}\left[\begin{array}{ll}1,08 & 1,76\end{array}\right]^{T}$.
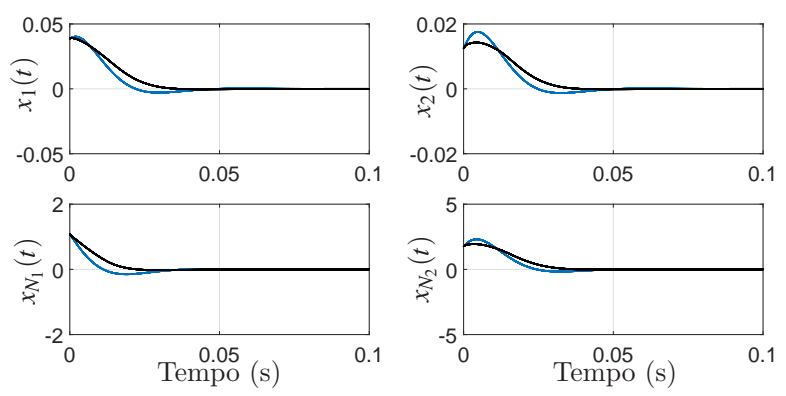

Figura 6. Variáveis de estado e variáveis de estado auxiliares $\left(x_{N}(t)=\left[\begin{array}{ll}\hat{x}(t)^{T} & u(t)^{T}\end{array}\right]^{T}\right)$ da simulação do sistema (1), com (40), Teorema 2 e Lema 2: com Teorema 1 , lei de controle (17) e (42), representados por (-); Corolário 1 , lei de controle $u_{N}(t)=-K x_{N}(t)$ e $(41)$, representado pela curva (-).

Nas Figuras de 5 a 7 , as curvas (-) representam as simulações utilizando o Corolário 1, Teorema 2 (tomando $K_{k}=K$ ) com o Lema 2, as curvas (-) representam as simulações utilizando os Teoremas 1 e 2 com o Lema 2.

Na Figura 5, a região elipsoidal para o caso do controle chaveado representado pela curva (-) é maior que a região obtida com o ganho constante, representado pela curva (-).

Na Figura 7, para o sistema (1), (39) e (40), considerando a condição inicial $x(0)=A_{2}^{-1}\left[\begin{array}{ll}1,08 & 1,76\end{array}\right]^{T}$, ou seja, $x_{N}(0)=\left[\begin{array}{lll}1,08 & 1,76 & 0\end{array}\right]^{T}$, a função de Lyapunov $V\left(x_{N}(0)\right)=$ $x_{N}(0)^{T} P x_{N}(0)=0,5367$ com matrix $P$ dada em (42). Para o sistema com controle robusto com ganho constante obtémse $V\left(x_{N}(0)\right)=x_{N}(0)^{T} P x_{N}(0)=0,9910$ com $P$ dada em (41).

\section{CONCLUSÕES}

A realimentação derivativa é adequada para implementações práticas nos quais a derivada do vetor de estado do sistema é mais fácil de se medir do que o vetor de estado do sistema. Em implementações reais, geralmente o sinal de controle está sujeito à saturação do atuador. Este manuscrito investigou o projeto de controle chaveado para sistemas lineares invariantes no tempo com incertezas politópicas e sujeito à saturação do atuador. Foi proposto
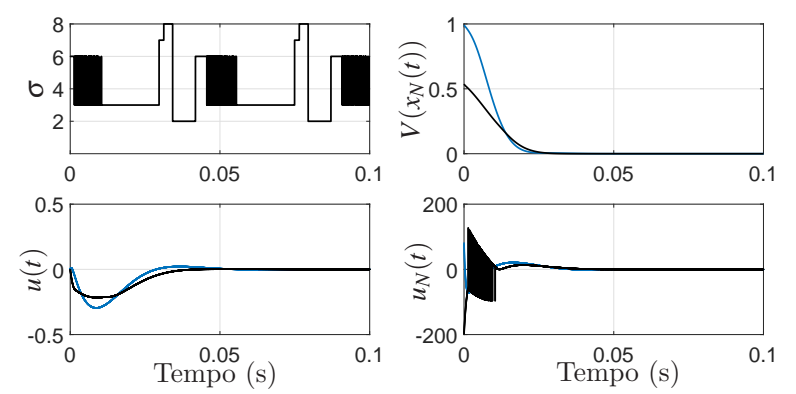

Figura 7. Índice de chaveamento $\sigma \in \mathbb{I}_{r}$, função de Lyapu$\operatorname{nov} V\left(x_{N}(t)\right)=x_{N}(t)^{T} P x_{N}(t)\left(x_{N}(t)=\left[\begin{array}{ll}\hat{x}(t)^{T} & u(t)^{T}\end{array}\right]^{T}\right)$, entrada de controle $u(t)=x_{N_{3}}(t)$ e sinal $u_{N}(t)$, para o sistema (1) e (40), Teorema 2 e Lema 2: com Teorema 1 , lei de controle (17) e (42), representados por (-); Corolário 1 , lei de controle $u_{N}(t)=-K x_{N}(t)$ e $(41)$, representado pela curva (-).

um projeto de controle chaveado baseado em uma dinâmica auxiliar, para plantas dadas em (1) e (2), sendo que $A(\alpha)$ deve ser uma matriz de posto completo e $B$ uma matriz constante, o que permitiu usar uma descrição convexa para a saturação do atuador. É importante notar que esta descrição para a saturação é válida em uma região de operação da dinâmica auxiliar e é garantida para todas as condições iniciais em um conjunto elipsoidal em que o estado permanece nesta região de operação para todo $t \geq 0$. No exemplo, mostrou-se a vantagem de se trabalhar com a lei de controle chaveada comparada com a lei de controle com ganho constante de realimentação.

\section{AGRADECIMENTOS}

Ao Conselho Nacional de Desenvolvimento Científico e Tecnológico - CNPq (Processo: 140131/2019-1 (Bolsa de doutorado)), (Processos: 309872/2018 - 9, 301227/2017 9, 306634/2017 - 1 (Bolsas de pesquisa)), a FAPESP (Processo: 2011/17610-0) e a Coordenação de Aperfeiçoamento de Pessoal de Nível Superior - Brasil (CAPES) Código de Financiamento 001.

\section{REFERÊNCIAS}

Abdelaziz, T.H.S. and Valášek, M. (2004). Pole-placement for SISO linear systems by state-derivative feedback. IEE Proceedings - Control Theory and Applications, 151, 377-385(8).

Abdelaziz, T.H.S. and Valášek, M. (2005). State derivative feedback by LQR for linear time-invariant systems. volume 38, 435 - 440. 16th IFAC World Congress.

Alves, U.N.L.T., Teixeira, M.C.M., Oliveira, D.R., Cardim, R., Assunção, E., and de Souza, W.A. (2016). Smoothing switched control laws for uncertain nonlinear systems subject to actuator saturation. International Journal of Adaptive Control and Signal Processing, 30(8-10), 1408-1433.

Assunção, E., Teixeira, M.C.M., Faria, F.A., da Silva, N.A.P., and Cardim, R. (2007). Robust state-derivative feedback LMI-based designs for multivariable linear systems. International Journal of Control, 80(8), 12601270 . 
Boyd, S., Ghaoui, L.E., Feron, E., and Balakrishnan, V. (1994). Linear matrix inequalities in system and control theory, volume 15. Siam.

Buzetti, A.S. (2017). Projeto de Controle Robusto Chaveado com Falhas nos Sensores. Mestrado, Universidade Estadual Paulista, Faculdade de Engenharia de Ilha Solteira.

Cao, Y.Y. and Lin, Z. (2003). Robust stability analysis and fuzzy-scheduling control for nonlinear systems subject to actuator saturation. IEEE Transactions on Fuzzy Systems, 11(1), 57-67.

Cardim, R., Teixeira, M.C.M., Assunção, E., and Covacic, M.R. (2007). Design of state-derivative feedback controllers using a state feedback control design. IFAC Proceedings Volumes, 40(20), 22-27. 3rd IFAC Symposium on System Structure and Control.

da Silva, E.R.P., Assunção, E., Teixeira, M.C.M., and Buzachero, L.F.S. (2012). Less Conservative Control Design for Linear Systems with Polytopic Uncertainties via State-Derivative Feedback. Mathematical Problems in Engineering, 2012, 1-21.

da Silva, E.R.P., Assunção, E., Teixeira, M.C.M., and Cardim, R. (2013). Robust controller implementation via state derivative feedback in an active suspension system. In 2013 Conference on Control and FaultTolerant Systems (Sys Tol), $752-757$.

da Silva, E.R.P., Assunção, E., Teixeira, M.C.M., Faria, F.A., and Buzachero, L.F.S. (2011). Parameterdependent Lyapunov functions for state-derivative feedback control in polytopic linear systems. International Journal of Control, 84(8), 1377-1386.

de Oliveira, D.R., Teixeira, M.C.M., Alves, U.N.L.T., de Souza, W.A., Assunção, E., and Cardim, R. (2018). On Local $\mathscr{H}_{\infty}$ Switched Controller Design for Uncertain T-S Fuzzy Systems Subject to Actuator Saturation with Unknown Membership Functions. Fuzzy Sets and Systems, 344, 1-26. Theme: Control Engineering.

Duan, Y.F., Ni, Y.Q., and Ko, J.M. (2005). Design guidelines for open-loop vibration control of stay cables using MR dampers. volume 5765, 667-689.

Efberg, J. and Löfberg, J. (2004). YALMIP : A Toolbox for Modeling and Optimization in MATLAB. In CACSD CONFERENCE, [s.n.], 2004, Taipei. Proceedings..., 284-289. Taipei.

Gahinet, P., Nemirovskii, A., Laub, A.J., and Chilali, M. (1994). The lmi control toolbox. In Proceedings of 1994 33rd IEEE Conference on Decision and Control, volume 3, 2038-2041.

$\mathrm{Hu}, \mathrm{T}$. and Lin, Z. (2003). Composite quadratic Lyapunov functions for constrained control systems. IEEE Transactions on Automatic Control, 48(3), 440-450.

$\mathrm{Hu}, \mathrm{T} ., \mathrm{Lin}, \mathrm{Z}$., and Chen, B.M. (2002). An analysis and design method for linear systems subject to actuator saturation and disturbance. Automatica, 38(2), 351 359 .

Klug, M., Castelan, E.B., and Coutinho, D. (2015). A T$\mathrm{S}$ fuzzy approach to the local stabilization of nonlinear discrete-time systems subject to energy-bounded disturbances. Journal of Control, Automation and Electrical Systems, 26(3), 191-200.

Moreira, M.R., Júnior, E.I.M., Esteves, T.T., Teixeira, M.C.M., Cardim, R., Assunção, E., and Faria, F.A. (2010). Stabilizability and disturbance rejection with state-derivative feedback. Mathematical Problems in Engineering, 2010, 1-12.

Moreira, M.R. (2015). Controle Robusto de Sistemas Não Lineares e Chaveados de Sistemas Lineares usando Realimentação Derivativa. Doutorado, Universidade Estadual Paulista, Faculdade de Engenharia de Ilha Solteira.

Reithmeier, E. and Leitmann, G. (2003). Robust vibration control of dynamical systems based on the derivative of the state. 72(11), 856-864.

Rossi, F.Q., Galvão, R.K.H., Teixeira, M.C.M., and Assunção, E. (2018). Direct discrete time design of robust state derivative feedback control laws. International Journal of Control, 91(1), 70-84.

Souza, W.A.d., Teixeira, M.C.M., Santin, M.P.A., Cardim, R., and Assunção, E. (2013). On switched control design of linear time-invariant systems with polytopic uncertainties. Mathematical Problems in Engineer, 2013, 110.

Wolmuth, L.D., Alves, U.N.L.T., Teixeira, M.C.M., Assunção, E., Cardim, R., and Moreira, M.R. (2019). Derivative feedback control for a class of uncertain linear systems subject to actuator saturation. Journal of Control, Automation and Electrical Systems, 30(4), 490-500.

Yazici, H. and Sever, M. (2017a). Design of an optimal state derivative feedback LQR controller and its application to an offshore steel jacket platform. An International Journal of Optimization and Control: Theories 8 Applications (IJOCTA), 8(1), 84-91.

Yazici, H. and Sever, M. (2017b). Output derivative feedback vibration control of an integrated vehicle suspension system. Proceedings of the Institution of Mechanical Engineers, Part I: Journal of Systems and Control Engineering, 1-11. 